\title{
Maintenir ses rêves bien vivants et prendre soin de soi
}

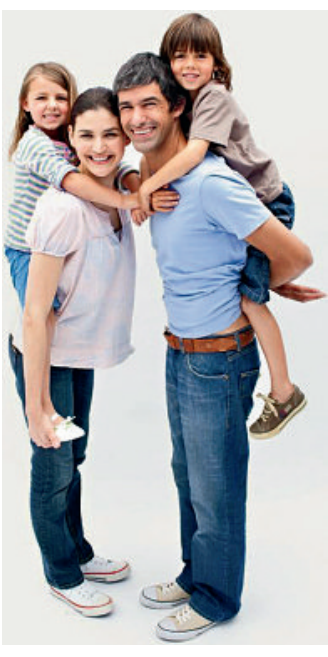

Pour une vie professionnelle réussie, les proches - et savoir donner attention à soi-même - jouent un rôle important.

1 Occasion bienvenue de rappeler le Programme ReMed mis en œuvre par la FMH pour venir en aide, de confrère à confrère, aux médecins en difficulté personnelle ou professionnelle (Hersperger M.

ReMed - un soutien au bon moment. Bull Méd Suisses. 2010;91(44):1717.

2 Resnick MD, Bowles G. Us and them: Worldwide health issues for adolescents. Lancet 2007; 369(9567):1058-60 Resnick MD. Best bets for improving the odds for optimum youth development. In: Kline KK (ed.). Authoritative Communities: The Scientific Case for Nurturing the Whole Child. New York: Springer; 2008. p. 137-150.
En janvier 2011, l'Institut universitaire de médecine sociale et préventive de Lausanne, dirigé par F. Paccaud après l'avoir été par F. Gutzwiller, fêtait son $40^{\text {e }}$ anniversaire; en effet, il a été créé par le Prof. Armand Delachaux à l'époque de l'émergence de cette branche - et de la santé publique - dans nos Facultés. Occasion de montrer comment, sur plusieurs créneaux, l'IUMSP s'est fait une place scientifique au plan international. Il a entre autres établi des ponts substantiels entre la «santé/médecine populationnelle» et la clinique: épidémiologie clinique, registre des cancers, maladies cardio-vasculaires, recherche sur les services de santé, etc. Ainsi qu'avec l'Unité multidisciplinaire de santé des adolescents (Prof. P.-A. Michaud).

Un keynote speaker était un spécialiste de l'adolescence de l'Université du Minnesota, Michael D. Resnick. Surprise (en 2011): sa remarquable présentation a été strictement ex cathedra, sans PowerPoint, avec un tour marqué d'éthique sociale voire de spiritualité. Il a parlé de ce dont les adolescents ont besoin pour devenir des adultes compétents et équilibrés; soulignant que, aujourd'hui dans un monde très complexe, «la santé des adolescents ne peut plus être la responsabilité des parents et des familles seulement» - idée que les milieux conservateurs rejettent alors que le besoin est avéré que l'école et d'autres apportent des contributions. Un changement des quinze ou vingt dernières années, c'est que les jeunes n'ont plus besoin des adultes - y compris des enseignants universitaires comme source première d'information. Notre rôle sera, dit Resnick, celui d'interprètes, afin d'aider nos successeurs à savoir quelle information est valable, et laquelle est utile. Il y a sur le web des informations exactes et d'autres inexactes et nous devons insister sans retenue sur ce qui est solidement démontré, par rapport à ce qui ne l'est pas.

Il importe de «maintenir nos rêves bien vivants, quelque chose que nous discutons rarement dans des réunions comme celle-ci. Si notre sentiment de plaisir, de joie, disparaît, il nous est très difficile d'œuvrer à guérir un monde abîmé qui a besoin de nos mains et de notre cœur.» La première chose est de prendre au sérieux l'amitié et la vie de famille; «j'espère que chacun d'entre nous est un bon père une bonne mère, un ami fiable.» Il faut oser dire que prendre soin de soi n'est pas égoïste, cela donne la force d'engager les combats qui doivent l'être. Ne pas croire qu'on n'est pas à la hauteur! La grande (sic)
Eleanor Roosevelt a dit: «Personne ne peut vous contraindre à vous sentir inférieur sans votre consentement.»

Comment faire pour ne pas être parfois submergés, dépassés: «Il s'agit de filtrer, de choisir entre ce que nous acceptons de laisser entrer en nous et ce que nous rejetons comme dangereux pour nous-mêmes et notre travail. Aussi, n'acceptons que ce que nous pouvons gérer.»

Quoi d'autre? Regarder en soi, respirer profondément au moins deux fois par jour (souvent - J. M. au cours de ma carrière avec beaucoup d'occasions de voir des gens désabusés, fatigués, ai-je fait une telle recommandation!). Et je pense ici à Ingrid Betancourt (impressionnante), à Lausanne à mi-février, appelant à «ne pas/ne plus se donner de bonnes raisons d'être malheureux». Si juste.

«Se reposer sur ses amis, trouver un endroit tranquille dans la nature où on peut se reconnecter avec ce sentiment de 〈révérence〉, de respect admiratif (awe), de merveille et de mystère.» Même quand des questions demandent en urgence nos bras et notre intelligence, prendre le temps de travailler sur soi. «Souvenez-vous comment votre visage est beau quand vous souriez. Prenez soin de votre corps. Parce que quand vous faites cela, les autres ressentent votre énergie, c'est comme une radio quand elle est sur la bonne fréquence, le bruit devient musique (...) $\mathrm{Ne}$ doutez jamais que vos actions vont faire une vraie différence.»

Au-delà d'un certain enthousiasme à l'américaine qu'on peut trouver parfois surfait, M. Resnick a apporté des messages majeurs: notre rôle vis-à-vis de ceux qui nous suivent a changé, notre érudition personnelle ne peut plus être, objectivement, ce qui nous fait supérieurs - mais (J. M.) notre consolation peut être que l'expérience elle ne s'apprend jusqu'ici ni dans les livres ni sur le web (jusqu'au moment où les progrès des neurosciences permettront de «transvaser» dite expérience des uns chez les autres?). Notre rôle d'enseignant, de tuteur, doit donner dans l'écoute, le coaching, la facilitation, la super/intervision, l'échange. Et puis, pour chacun, prendre soin de soi. Pour maintenir à distance le burn out, l'épuisement. Pour garder le plaisir de travailler - et aussi, de temps en temps, de ne pas travailler $[1,2]$.

Jean Martin, membre de la Commission nationale d'éthique et de la rédaction du BMS 